\title{
Comparison of the Previous and Current Trauma-Related Shock Classifications: A Retrospective Cohort Study from a Level I Trauma Center
}

\author{
Péter Jávor ${ }^{\mathrm{a}}$ Endre Csonka ${ }^{\mathrm{a}}$ Edina Butt ${ }^{\mathrm{a}}$ Ferenc Rárosi $^{\mathrm{b}}$ Barna Babik $^{\mathrm{c}}$ \\ László Török $^{a}$ Endre Varga ${ }^{a}$ Petra Hartmann ${ }^{d}$

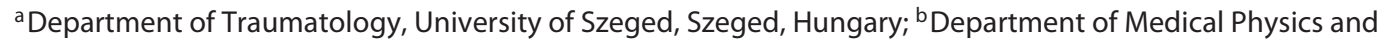 \\ Informatics, University of Szeged, Szeged, Hungary; ' Department of Anaesthesiology and Intensive Therapy,

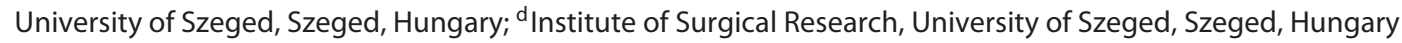

\section{Keywords}

Multiple trauma $\cdot$ Shock, hemorrhagic · Vital signs · Heart rate $\cdot$ Advanced Trauma Life Support program

\begin{abstract}
Purpose: The aim was to examine the predictive value of the hypovolemic shock classification currently accepted by the Advanced Trauma Life Support (ATLS) program over the previous one, which used only vital signs (VS) for patient allocation. The primary outcome was 30-day mortality; as secondary outcome, heart rate (HR), systolic blood pressure (SBP), Glasgow Coma Scale (GCS) and base deficit (BD) data were compared and investigated in terms of mortality prediction. Methods: Retrospective analysis at a level I trauma center between 2014 and 2019. Adult patients treated by trauma teams were allocated into severity classes (I-IV) based on the criteria of the current and previous ATLS classifications, respectively. The prognostic values for the classifications were determined with Fisher's exact test and $X^{2}$ test for independence, and compared with the 2-proportion $Z$ test. The individual variables were analyzed with receiver-operating characteristic (ROC) analyses. Results: A total of 156 patients met the inclusion criteria. Mortality was effectively predicted by both classifications, and there was no statistically significant difference
\end{abstract}

between the predictive performances. According to ROC analyses, GCS, BD and SBP had significant prognostic values while HR change was ineffective in this regard. Conclusions: The currently used ATLS shock classification does not appear to be superior to the VS-based previous classification. GCS, BD and SBP are useful parameters to predict the prognosis. Changes in HR do not reflect the clinical course accurately; thus, further studies will be needed to determine the value of this parameter in trauma-associated hypovolemic-hemorrhagic shock conditions.

(c) 2021 The Author(s) Published by S. Karger AG, Basel

\section{Introduction}

\section{Background}

Blood loss is one of the leading causes of death, but the most frequent single cause is still traumatic brain injury $[1,2]$. Hypovolemic shock is the second main cause of mortality in trauma patients [3,4], and the outcome can be improved significantly through early recognition, intravenous fluid resuscitation and blood transfusions [5]. Currently, the Advanced Trauma Life Support (ATLS) program provides well-established guidances for early assessment and initial management of major trauma and

Correspondence to:

Petra Hartmann, hartmann.petra@ med.u-szeged.hu

karger@karger.com www.karger.com/esr

Karger $\stackrel{\text { ' }}{5}$

GOPEN ACCESS
(C) 2021 The Author(s)

Published by S. Karger AG, Basel

This is an Open Access article licensed under the Creative Commons Attribution-NonCommercial-4.0 International License (CC BY-NC) (http://www.karger.com/Services/OpenAccessLicense), applicable to the online version of the article only. Usage and distribution for commercial purposes requires written permission. 
suspected hemorrhage by allocating the patients into severity classes I-IV, respectively $[6,7]$. Until recently, the earlier guidance proposed the use of vital signs (VS) only, including heart rate (HR), systolic blood pressure (SBP) and Glasgow Coma Scale (GCS) to aid the recognition of shock and estimate blood loss $[8,9]$. However, the predictive value of VS-based classification has been questioned, and in 2013, the analysis of the Trauma Register DGU ${ }^{\circledR}$ (Deutsche Gesellschaft für Unfallchirurgie) indicated that the VS-based classification does not reflect the clinical condition accurately. Therefore, the use of base deficit (BD) as a sole parameter in the classification has been recommended $[10,11]$.

$\mathrm{BD}$ is a metabolic marker reflecting the acid-base status of the patients and is commonly used to assess hemorrhage and its consequences [12-14]. Several studies have documented its ability to predict mortality and postinjury transfusion requirements and highlighted its role in the early assessment of injury severity of trauma patients [11, 15-19]. Therefore, the latest ATLS recommendation expanded the assessment criteria with the BD value [6]. However, the specificity of $\mathrm{BD}$ for hypovolemia prediction is still questionable, since not only metabolic acidosis, but other factors such crystalloids (lactated Ringer or saline) can also elevate BD [18]. Furthermore, alcohol or drugs, commonly seen in trauma patients, may also diminish its predictive accuracy [20,21]. Besides, it has been demonstrated that patients over 55 years may have significant injuries and mortality risk without manifest $\mathrm{BD}$ alterations [22].

\section{Objectives}

Based on this background, our primary goal was to examine the predictive power of the currently accepted ATLS classification of hypovolemic shock to confirm or refute the superiority of VS + BD detection over the previously used (VS only) protocol. For this purpose, we conducted a retrospective cohort analysis at a level I trauma center to compare the VS + BD and VS classifications in terms of their ability to predict mortality. Our secondary goal was to determine the parameters with strong prognostic possibilities in the early assessment phases of the injured. Therefore, the predictive values of HR, SBP, GCS and BD were also calculated.

\section{Materials and Methods}

\section{Study Design}

The present study is a retrospective cohort analysis at a single, level I trauma center located in an urban area. The level I qualifica- tion is based on national standards regarding the kinds of resources available and the number of patients admitted yearly. The designation criteria correspond to US standards.

\section{Data Collection}

In the past decades, there were several important changes in emergency trauma guidelines, such as the paradigm shift in fluid resuscitation and the introduction of tranexamic acid [23, 24]. Taking this into account, we decided to analyze data only from the past 5 years; the data were collected between July 11, 2014, and September 11, 2019, from the electronic database (MedSolution) at the University of Szeged. The protocols of emergency trauma care including massive transfusion protocols at the institution have been in compliance with the principles of ATLS during the whole study period.

\section{Inclusion Criteria}

The inclusion criteria consisted of trauma team activation, transport directly from scene, age of 16 years or greater, a detailed documentation including Abbreviated Injury Scale, Injury Severity Score, the accurate mechanism of injury and a complete data set for HR, SBP, GCS and BD recorded at presentation. Regarding physiological variables, the values of the first in-hospital measurements were included and reported. The activation of the trauma team is based on anatomical and physiological criteria and the mechanism of injury. High-energy trauma is characterized by a high amount of kinetic energy inducing severe tissue damage and typically sustained by road traffic accidents, crush or blast injuries, and falling from heights. The clinical handover between paramedics and emergency department staff follows the MIST and AMPLE templates (MIST: M - mechanism of injury, I - injuries sustained, $\mathrm{S}$ - signs, $\mathrm{T}$ - treatment and trends in the vital signs; AMPLE: A - allergies, $\mathrm{M}$ - medications, $\mathrm{P}$ past medical history, $\mathrm{L}$ - last ate, $\mathrm{E}$ - events). The age limit of 16 years was selected according to the fact that normal values of HR and SBP by adolescents above that age do not differ largely from the normal values by adults $[25,26]$.

The recorded variables included the mechanism of injury, the International Statistical Classification of Diseases and Related Health Problems (ICD) codes, vital parameters (HR, SBP and GCS) measured by the trauma team at presentation, BD and 30day survival. Although prehospital treatment might have influenced the parameters, paramedics use a unified protocol including guidance regarding the prehospital fluid resuscitation, administration of vasopressors and opioid analgesics also. Vasopressors have a controversial role in the management of the severely injured [27, 28]. They are administered mainly to maintain cerebral perfusion pressure in cases of severe traumatic brain injury and concomitant hemorrhage, where increasing intracranial pressure and decreasing mean arterial pressure work simultaneously against cerebral perfusion pressure. Another indication for using vasopressors is the failure of maintaining blood pressure with fluid resuscitation only $[28,29]$.

When patients were discharged from the hospital earlier than 30 days, the follow-up was completed as a part of outpatient care. Patients who received cardiopulmonary resuscitation on scene or primary survey in another institute were excluded. Additionally, imprecise documentation and missing variables also entailed exclusion. 
Table 1. Simplified shock severity classification using absolute values

\begin{tabular}{cclll}
\hline $\begin{array}{c}\text { Physiological variables } \\
\text { Estimated blood loss: }\end{array}$ & $\begin{array}{l}\text { Class I } \\
<15 \%\end{array}$ & $\begin{array}{l}\text { Class II } \\
15-30 \%\end{array}$ & $\begin{array}{l}\text { Class III } \\
31-40 \%\end{array}$ & $\begin{array}{l}\text { Class IV } \\
>40 \%\end{array}$ \\
\hline $\mathrm{VS}$ & & & & \\
$\mathrm{HR}^{1}, \mathrm{bpm}$ & $<100$ & $100-119$ & $120-139$ & $\geq 140$ \\
$\mathrm{SBP}^{1}, \mathrm{~mm} \mathrm{Hg}$ & $\geq 110$ & $100-109$ & $90-99$ & $<90$ \\
$\mathrm{GCS}^{1}$ & 15 & 15 & $12-14$ & $<12$ \\
$\mathrm{BD}^{2}, \mathrm{mEq} / \mathrm{L}$ & $0-2$ & $2-6$ & $6-10$ & $\geq 10$ \\
Transfusion & monitor & possible & yes & massive transfusion
\end{tabular}

The table is based on the recommendations of ATLS [6]. Pulse pressure, respiratory rate and urinary output are not included in the physiological variables. Instead of trends, absolute values were used to make the criteria more objective. VS, vital sign; HR, heart rate; SBP, systolic blood pressure; GCS, Glasgow Coma Scale; BD, base deficit; bpm, beats per minute. ${ }^{1}$ The current ATLS classification for hypovolemic shock only offers exact values for BD. The values for HR were adopted from the previous (9th) ATLS shock classification. For SBP and GCS, we adopted the values used by Dunham et al. [19]. ${ }^{2}$ A negative base excess is called BD and indicates metabolic acidosis.

\section{Patient Groups}

The ATLS does not explicitly declare whether the worst parameter or a combination of all the parameters should determine the severity class of the patient. Most trauma patients cannot be allocated correctly to the 4 ATLS severity classes (I-IV) when a combination of vital parameters is assessed $[8,10]$. Therefore, the participants' allocation was based on their worst parameter within the VS and VS + BD criteria. Since the current ATLS shock classification does not describe exact values for HR, SBP and GCS, we adopted HR values from the previous ATLS classification and SBP and GCS values from the study of Dunham et al. [19] to make the criteria objective and transparent (Table 1).

\section{Outcomes}

As a primary outcome, we compared the VS and VS + BD classifications with respect to 30 -day mortality, taking the close relationship of the severity of bleeding to the risk for adverse outcomes into consideration $[8,11,17,19,30]$.

As a secondary goal, we studied the prognostic potential for the individual parameters (HR, SBP, GCS and BD, respectively) to be able to determine the strongest and weakest predictive factors in the initial assessment.

\section{Statistical Analysis}

Continuous data were expressed as means \pm SD. Categorical data were expressed as frequency or relative frequency (percentages). $\chi^{2}$ tests for independence were performed to test the relationship between the VS + BD classification result and outcome of mortality. The assumption of the $\chi^{2}$ test for independence was slightly violated in the crosstabulation of the VS classification result and outcome of mortality, therefore Fisher's exact test was used to test the relationship between the VS classification result and outcome of mortality.

The 2-proportion $\mathrm{Z}$ test was performed to compare the predictive power of the VS and VS + BD classifications. Binary logistic regression was applied for further analysis between the VS + BD classification result (groups 1, 2 vs. groups 3,4) and outcome of mortality; odds ratio and $95 \%$ confidence interval (CI) for odds ratio were calculated.

Comparison of Trauma-Related Shock

Classifications

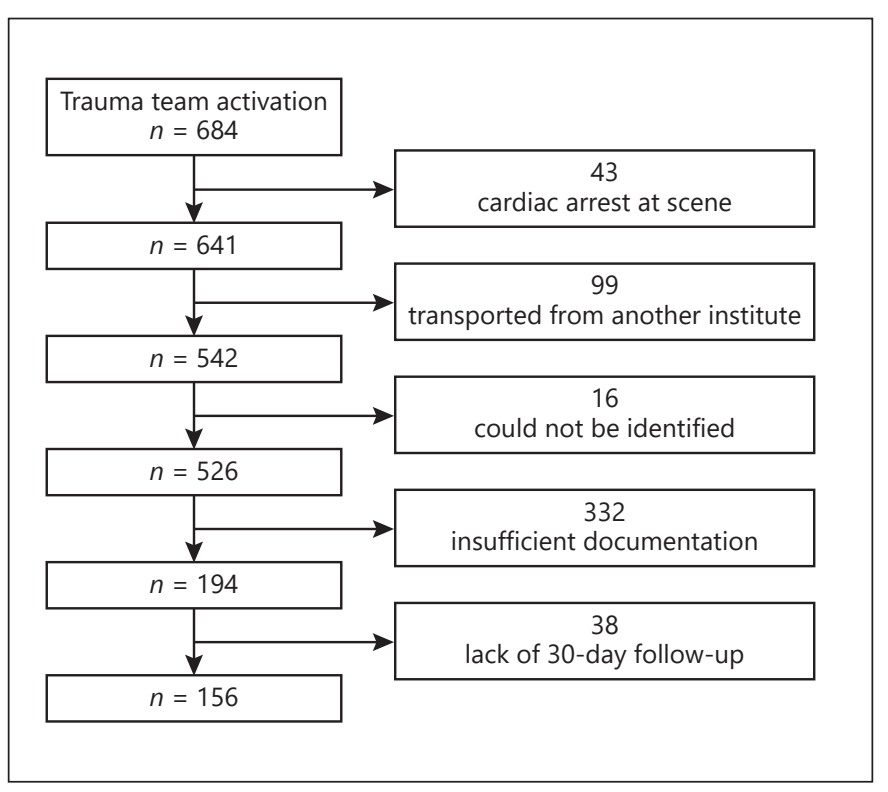

Fig. 1. Study flowchart. The study flowchart illustrates that 684 trauma team activations occurred during the reported period. After excluding patients who were in cardiac arrest at the scene of accident or primary survey in another institute, there were 542 patients left. Sixteen patients with detailed medical record could not be identified due to the lack of personal data. In 332 cases, there were either missing variables (HR/SBP/GCS/BD), or Injury Severity Score, Abbreviated Injury Scale or the accurate mechanism of injury were not recorded. A lack of 30-day follow-up occurred in 38 cases. Ultimately, 156 patients were enrolled in the final analysis.

The predictive performance of individual variables was assessed using receiver-operating characteristic (ROC) analysis. Area under the ROC curve (AUC) was calculated for each individual variable (candidate predictors: GCS, HR, SBP, BD). Hypothesis tests for AUC ROC were performed and 95\% confidence 
Table 2. Patient characteristics

\begin{tabular}{|c|c|c|c|c|c|c|c|c|c|}
\hline \multirow[t]{2}{*}{ Characteristic } & \multirow[t]{2}{*}{ All classes } & \multicolumn{2}{|l|}{ Class I } & \multicolumn{2}{|l|}{ Class II } & \multicolumn{2}{|l|}{ Class III } & \multicolumn{2}{|l|}{ Class IV } \\
\hline & & VS & $V S+B D$ & VS & $V S+B D$ & VS & $\mathrm{VS}+\mathrm{BD}$ & VS & $V S+B D$ \\
\hline Age (mean $\pm S D)$, years & $49.4 \pm 20.7$ & $48.0 \pm 18.7$ & $46.4 \pm 15.2$ & $39.7 \pm 14.8$ & $48.2 \pm 19.9$ & $47.0 \pm 24.3$ & $44.2 \pm 23.9$ & $52.1 \pm 21.2$ & $52.2 \pm 21.3$ \\
\hline Female, \% & 26.9 & 30.6 & 33.3 & 20.0 & 23.3 & 23.8 & 26.1 & 26.3 & 26.6 \\
\hline Male, \% & 73.1 & 69.4 & 66.6 & 80.0 & 76.7 & 76.2 & 73.9 & 73.7 & 73.4 \\
\hline $\mathrm{HR}($ mean $\pm \mathrm{SD}), \mathrm{bpm}$ & $82.3 \pm 21.4$ & $78.0 \pm 11.1$ & $79.3 \pm 9.4$ & $90.7 \pm 21.3$ & $81.5 \pm 15.5$ & $88.0 \pm 18.5$ & $84.4 \pm 20.5$ & $82.4 \pm 26.4$ & $82.9 \pm 26.0$ \\
\hline $\mathrm{SBP}($ mean $\pm \mathrm{SD}), \mathrm{mm} \mathrm{Hg}$ & $125.7 \pm 33.5$ & $142.4 \pm 22.5$ & $144.2 \pm 22.1$ & $119.5 \pm 16.2$ & $137.0 \pm 23.9$ & $130.0 \pm 27.8$ & $127.4 \pm 22.7$ & $114.5 \pm 37.9$ & $115.3 \pm 38.3$ \\
\hline $\mathrm{GCS}($ mean $\pm \mathrm{SD})$ & $9.8 \pm 5.5$ & $15.0 \pm 0.0$ & $15.0 \pm 0.0$ & $15.0 \pm 0.0$ & $15.0 \pm 0.0$ & $13.7 \pm 1.0$ & $14.0 \pm 1.0$ & $4.7 \pm 3.3$ & $5.0 \pm 3.6$ \\
\hline $\mathrm{BD}($ mean $\pm \mathrm{SD}), \mathrm{mmol} / \mathrm{L}$ & $4.1 \pm 4.9$ & $2.2 \pm 2.1$ & $0.6 \pm 1.0$ & $3.3 \pm 3.6$ & $3.0 \pm 1.7$ & $4.7 \pm 5.6$ & $3.9 \pm 3.2$ & $5.3 \pm 5.6$ & $5.7 \pm 6.0$ \\
\hline Vasopressor need, $n$ (\%) & $36(23.1)$ & $2(4.1)$ & $0(0)$ & $0(0)$ & $1(3.3)$ & $5(23.8)$ & $5(21.7)$ & $29(38.2)$ & $30(38.0)$ \\
\hline
\end{tabular}

HR, heart rate; SBP, systolic blood pressure; GCS, Glasgow Coma Scale; BD, base deficit; SD, standard deviation; VS, vital sign. Slightly declining tendency in mean SBPs, increasing in mean BDs. A large decrease between mean GCS rates of classes II, III and IV, suggesting that GCS might have had the strongest influence on patient allocation. Need for vasopressors occurred mainly in classes III and IV.

bounds for AUC ROC were calculated with a nonparametric method. A $p$ value $<0.05$ was considered to be statistically significant. All data were analyzed by using statistical software IBM SPSS 25.0 (IBM Corporation, Chicago, IL, USA).

\section{Results}

\section{Patient Population and Demographics}

A total of 684 patients were admitted to our institution with activation of the trauma team. Ultimately, 156 participants met our inclusion criteria. The flowchart for patient enrollment is presented in Figure 1.

The mean age of the participants was $49.4 \pm 20.7$ years, and only $26.7 \%$ of the patients were female. The most common mechanisms of injury were road traffic accidents (56.4\%) and falls (29.5\%). The most affected body regions were the head and neck (74.4\%), thorax (53.9\%) and extremities (48.1\%). Due to the fact that most patients suffered a high energy trauma, multiple body regions were involved in several cases. The characteristics of the patient population are shown in Table 2; the distribution of injury mechanisms, affected body regions and clinical outcomes are demonstrated in Table 3.

\section{Results of Statistical Analyses}

According to VS, 31.4\% of the patients were assigned to class I, $6.4 \%$ to class II, $13.5 \%$ to class III and $48.7 \%$ to class IV. Based on VS + BD criteria, $16.0 \%$ of the patients were reallocated to a higher severity class; however, this change affected mostly the low-risk classes (I and II). Thirty-four patients died within the first 30 days, resulting in a mortality rate of $21.8 \%$. The distribution of pa- tients and mortality among the classes are shown in Figure 2.

Both the VS and VS + BD classifications showed a strong relation to mortality ( $\chi^{2}$ and Fisher's exact tests $p_{\mathrm{VS}}=0.0001$ vs. $p_{\mathrm{VS}+\mathrm{BD}}=0.000009$, respectively). These results are shown in Tables 4 and 5. According to the 2 -proportion $\mathrm{Z}$ test, there was no significant difference in their predictive performance of mortality $(p=0.9808)$.

Through a separate analysis of HR, SBP, GCS and BD, we found that GCS has the highest prognostic power $\left(\mathrm{AUC}_{\mathrm{GCS}}=0.799, p<0.001 ; \mathrm{CI}=0.722-0.875\right)$. Derangements in $\mathrm{BD}$ and $\mathrm{SBP}$ were significant but weak predictors of mortality $\left(\mathrm{AUC}_{\mathrm{BD}}=0.683, p=0.001, \mathrm{CI}=0.576-\right.$ $\left.0.790 ; \mathrm{AUC}_{\mathrm{SBP}}=0.633, p=0.018, \mathrm{CI}=0.521-0.744\right)$. HR was found ineffective in prognosis $\left(\mathrm{AUC}_{\mathrm{HR}}=0.595, p=\right.$ $0.090, \mathrm{CI}=0.480-0.710$ ). The results of the ROC analysis with the ROC curves for the variables are shown in Figure 3.

The binary logistic regression analysis confirmed that the risk for mortality increases massively in the higher severity classes (III and IV) as compared to the less severe ones (I and II). The results of the analysis are demonstrated in online supplementary Table 1 (for all online suppl material, see www.karger.com/doi/10.1159/000516102).

\section{Discussion}

\section{Interpretation of Results}

This study was designed to investigate the previous, VS-based and the current ATLS classifications and the prognostic power of the parameters. Both classifications were highly effective in predicting mortality, with no sig- 
Table 3. Severity and mechanisms of injury, affected body regions, outcomes

\begin{tabular}{ll}
\hline & Patient \\
population \\
$(n=156)$
\end{tabular}

nificant difference between their prognostic values. Therefore, the superiority of VS + BD over the VS classification could not be confirmed.

Some other findings are also noteworthy, as we have demonstrated that more than $90 \%$ of all deaths were distributed in classes III and IV. This underlines the importance of the threshold between classes II and III, where the first derangements in SBP, respiratory rate and urinary output usually occur [6]. According to other studies, the threshold $\mathrm{BD}$ value between these 2 classes $(6 \mathrm{mmol} / \mathrm{L})$ shows a notable predictive potential for mortality and

Pelvic or sacral fracture

Isolated pubic ramus fracture

$25(16.0)$

Isolated iliac wing fracture

$6(24.0)$

Isolated sacral fracture

$1(4.0)$

Open book injury

$2(8.0)$

Lateral compression injury

Unilateral complete posterior disruption

Extremities

Shoulder or upper arm

Elbow or forearm

$4(16.0)$

$11(44.0)$

$1(4.0)$

$75(48.1)$

$23(30.7)$

$19(25.3)$

Wrist or hand

$14(18.7)$

Hip or thigh

$26(34.7)$

$35(46.7)$

Ankle or feet

$6(8.0)$

Spine

Fracture of the cervical spine

$40(25.6)$

$4(10.0)$

Fracture of the thoracal spine

$11(27.5)$

Fracture of the lumbar spine

$25(62.5)$

\section{Outcomes, $n$ (\%)}

Administration of blood products

$97(62.2)$

Massive transfusion

$27(17.3)$

Administration of vasopressors

$36(23.1)$

Need for ICU admission

Surgery in the first $24 \mathrm{~h}$

$111(71.2)$

Mortality in the first $24 \mathrm{~h}$

$89(57.1)$

Mortality in the first 30 days

$16(10.3)$

$34(21.8)$

ISS, Injury Severity Score; AIS, Abbreviated Injury Scale; IQR, interquartile range; ICU, intensive care unit. AIS coding was performed based on the AIS 2008 dictionary. Road traffic accidents and falls were the most common mechanisms that required the activation of the trauma team. The regions of the head and neck, thorax and extremities were involved in a high number of cases. More than half of the participants underwent surgery in the first $24 \mathrm{~h}$. Blood products were administered in 97 cases. 34 patients died within 30 days, resulting in a mortality rate of $21.8 \%$.

transfusion requirements after injury [16, 22, 31, 32]. Six millimoles per liter is also the threshold from where the administration of blood products is recommended by ATLS [6]. The therapeutic and prognostic relevance of this point questions the reasonability of dividing trauma patients into 4 different severity groups. Additionally, due to the need for rapid decisions in the emergency trauma setting, the complexity of the ATLS shock classification has already received criticism before its extension with the $\mathrm{BD}$ [33]. Based on our study, combining the less severe classes (I and II) and the severe classes (III and IV) could be a le- 

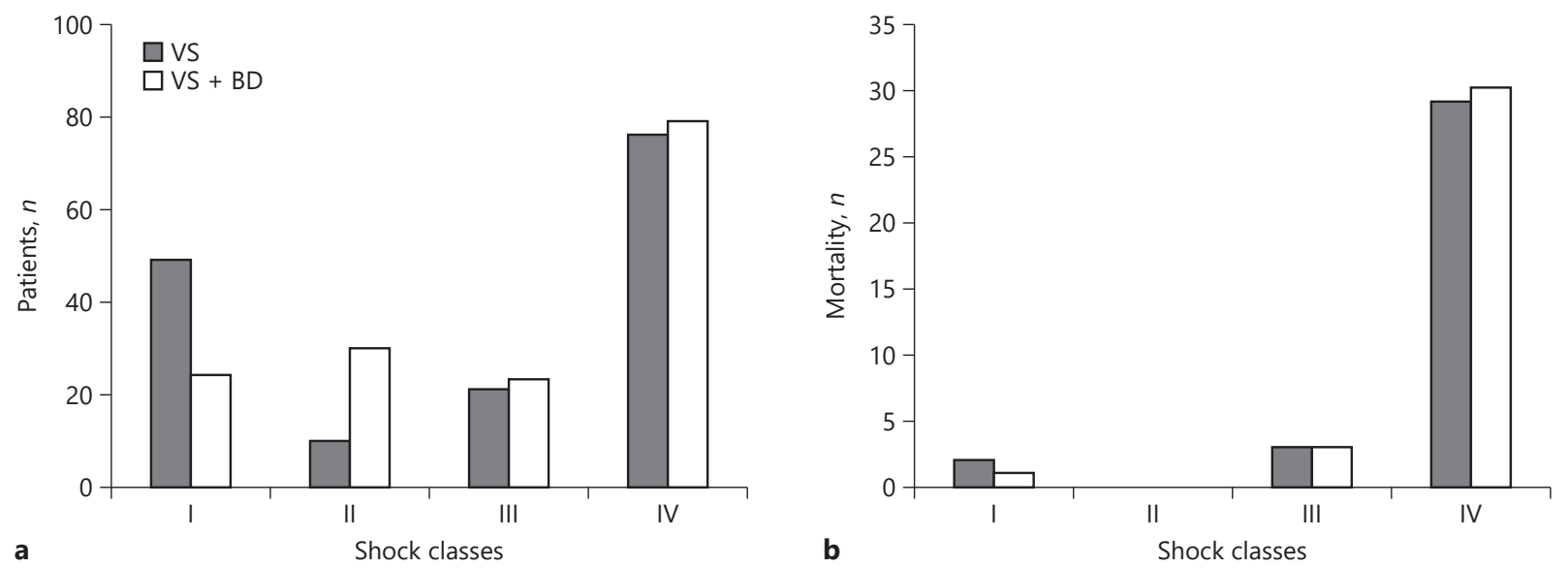

Fig. 2. Distribution of patients (a) and mortality (b) among the shock severity classes based on VS and VS + BD. The difference in patient allocation mostly occurred in low-risk classes (I and II). Diagram a suggests that BD was not a key parameter in determining the severity class. Diagram $\mathbf{b}$ shows that the vast majority of mortality cases is located in class IV.

Table 4. Fisher's exact test of vital signs (VS) and mortality

\begin{tabular}{lllll}
\hline Variables & Shock classes & Survival & Exitus & Total \\
\hline HR; SBP; GCS & I & 47 & 2 & 49 \\
& II & 10 & 0 & 10 \\
& III & 18 & 3 & 21 \\
Total & IV & 47 & 29 & 76 \\
& & 122 & 34 & 156 \\
\hline
\end{tabular}

Fisher's exact test $p$

0.000

value

The results demonstrate a strong relation between mortality and VS classification. A $p$ value $<0.001$ was considered to be statistically significant. Survival and death refer to 30-day mortality. HR, heart rate; SBP, systolic blood pressure; GCS, Glasgow Coma Scale.

gitimate option to increase the practicality of the classification. A pragmatic scale for the assessment of hemorrhage in decision-making and triage may reduce the time from patient arrival to massive transfusion protocol activation and involvement of the right specialist (e.g., abdominal surgeon, thoracic surgeon, etc.). Of course, a simplified classification like this should always be evaluated together with the adjuncts of the primary survey (e.g., extended focused assessment with sonography for trauma and pelvic $\mathrm{X}$-ray). During the secondary survey, trauma patients could undergo a comprehensive, detailed assessment to estimate the extent of optimal fluid replacement.
Table 5. $x^{2}$ test of VS $+B D$ and mortality

\begin{tabular}{lllll}
\hline Variables & Shock classes & Survival & Exitus & Total \\
\hline HR; SBP; GCS; BD & I & 23 & 1 & 24 \\
& II & 30 & 0 & 30 \\
& III & 20 & 3 & 23 \\
Total & IV & 49 & 30 & 79 \\
\hline \multicolumn{2}{l}{} & 122 & 34 & 156 \\
\hline Pearson $x^{2}$ & df & \multicolumn{3}{l}{$p$ value } \\
\hline
\end{tabular}

$\mathrm{HR}$, heart rate; SBP, systolic blood pressure; GCS, Glasgow Coma Scale; BD, base deficit; $d f$, degrees of freedom; VS, vital sign. The results demonstrate a strong relation between mortality and VS + BD classification. A $p$ value $<0.001$ was considered to be statistically significant. Survival and death refer to 30-day mortality.

It is important to note that we investigated ATLS shock classifications as early prognostic tools in the primary survey, where the exact set of injuries is unknown. We took it into consideration that the adequate evaluation of traumatic brain injuries can only be accomplished with computer tomography, after the primary survey. Therefore, the 23 patients who sustained isolated head injuries were not excluded from our study.

As a secondary outcome, the predictive values of the individual variables were evaluated. GCS, BD and SBP 
showed a significant predictive performance. While GCS displayed a relatively strong relation to the outcome, the relation was weak for BD and SBP. In our study, BD and SBP alone did not appear to have a sufficiently high prognostic potential to be the foundation for the early assessment. According to other studies, SBP is considered to have a poor reliability in the early assessment, since hypotension usually does not occur until the degree of shock is profound $[3,34]$. Despite of its strong relation to mortality, the GCS can be affected by several factors besides hypotension, such as traumatic brain injury or alcohol intoxication [35].

Of note, HR did not have a significant relation to mortality in our study. Numerous factors such as anxiety, pain and medication can lead to elevated HR, making the specificity of tachycardia for hypotension questionable [35, 36]. Increased HR may also be masked via $\beta$-blockers [37, 38] (particularly in combination with $\mathrm{Ca}^{2+}$ channel inhibitors and angiotensin-converting enzyme inhibitors) or physiological bradycardia [39]. Multiple studies pointed out that HR tends to demonstrate a biphasic response to bleeding since the patients develop bradycardia as blood loss becomes profound after initial tachycardia [34, $40,41]$. In our study, the predictive values of the individual variables showed the following ranking: GCS $>\mathrm{BD}>$ $\mathrm{SBP}>\mathrm{HR}$. The relevant differences between the variables suggest that weighing them and using their combination to allocate trauma patients could potentially increase the accuracy and specificity of the classification for hemorrhage. However, further research with larger sample sizes is required to elaborate such modifications.

\section{Limitations}

The retrospective nature can be considered as a limitation in itself. Although our cohort analysis with the given sample size only has limited value as a validation study, it provides original research information in a controversial topic and highlights potential areas of improvement for an assessment tool that is mistrusted by several clinicians [8].

With regard to GCS upon admission, the concern emerges whether prehospital intubation had a major influence on patient allocation. In accordance with the paramedics protocol, on-scene intubation is indicated in case of GCS $<8$, which already entails the allocation into class IV according to the criteria of shock classification used in our study. Consequently, the distortional effect of prehospital intubation on our results is not likely.

The use of vasopressors occurred mostly in classes IIIIV, thus having less influence on our results. The notable amount of excluded patients and our inability to include

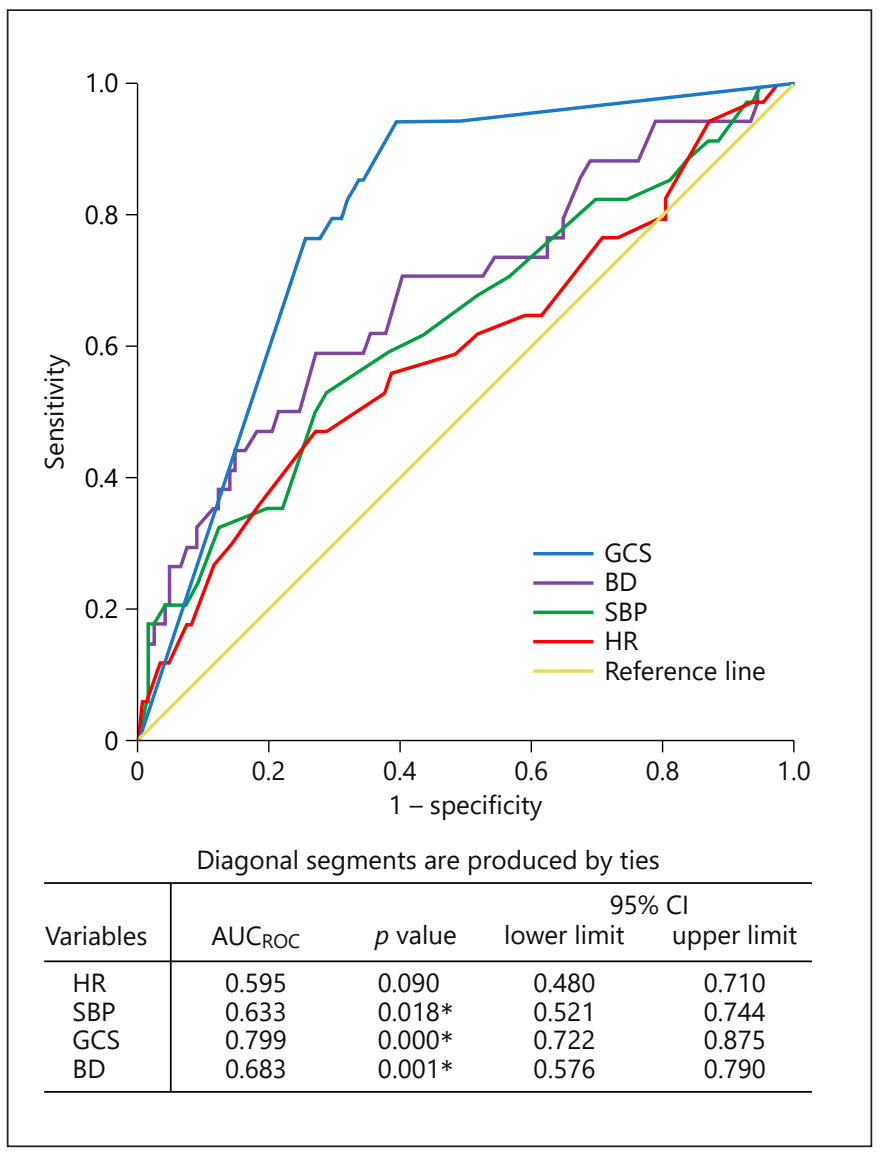

Fig. 3. ROC analysis of the individual variables. HR, heart rate; SBP, systolic blood pressure; GCS, Glasgow Coma Scale; BD, base deficit; $\mathrm{AUC}_{\mathrm{ROC}}$, area under the receiver-operating characteristic curve. ROC curves for the individual parameters. GCS has the largest $\mathrm{AUC}_{\mathrm{ROC}}$, showing the superiority of its predictive value over the other variable. A $p$ value $<0.05$ was considered to be statistically significant. ${ }^{*} p<0.05$.

respiratory rate and urinary output in the analysis are due to the lack of comprehensive documentation.

\section{Conclusions}

Despite the significant relationship between $\mathrm{BD}$ and mortality, the previous and current ATLS classifications yielded nearly equivalent predictive performances, thereby rendering the added value of $\mathrm{BD}$ to the classification questionable. Undoubtedly, postinjury VS values are continuously changing during trauma care and individual reactions can vary greatly. Despite these facts, trends may appear in the derangements of physiological variables. In this study, the capability of individual variables to predict 
mortality followed the GCS $>$ BD $>$ SBP $>$ HR ranking. The role of HR in the early assessment of trauma patients may be worth reconsideration in further studies since it does not seem to reflect the clinical condition accurately. Although the ATLS shock classification is not necessarily a pragmatic scale, it has become taught in more than 60 countries, making an impact on the approach of young clinicians to the initial management of the severely injured. Taking these considerations into account, striving for further targeted clinical investigations and the development of the classification may be advantageous.

\section{Acknowledgment}

The authors are thankful for the critical remarks of Mihály Boros during planning the study design and preparation of the manuscript.

\section{Statement of Ethics}

The study was conducted in accordance with the Declaration of Helsinki and has been approved by the local medical ethics committee at the University of Szeged (Regional Human Biomedical Research Ethics Committee, University of Szeged; chairman: Prof. Dr. Tibor Wittmann) under reference No. 182/2019-SZTE. Given the retrospective nature of our study, no interventions have been performed. Upon admission, patients (or their relatives or guardians) signed a general informed consent including the permission to use their scientific information (except from personal data) for research purposes. The consent is available from: https://u-szeged. $\mathrm{hu} / \mathrm{szakk} / \mathrm{v} /$ adatvedelem. The present record does not include personal data, photographs or other material that might identify the participants of our research. Personal data were not given out and will not be given out without the consent of our patients.

\section{Conflict of Interest Statement}

The authors declare that they have no competing interests.

\section{Funding Sources}

The study was funded by National Research Development and Innovation Office grant NKFI K120232 and FK 138839. It was further funded by the Hungarian Academy of Sciences, János Bolyai Research Grant BO/00644/21.

\section{Author Contributions}

P.J.: preparation of the draft of the manuscript, formatting the manuscript, substantial contribution in study design, data extraction; E.C.: expert in the field of traumatology, validation of data extraction, interpretation of data; E.B.: data extraction, preparation of the standardized data collection sheet; L.T. and E.V.: experts in the field of traumatology, provided revisions to the scientific content of the manuscript; F.R.: statistical analyses; B.B.: expert in the field of anesthesiology and intensive therapy, provided revisions to the scientific content of the manuscript; P.H.: study design, study coordination, preparation of the manuscript, stylistic revision of the manuscript, provided revisions to the scientific content of the manuscript. Hereby, all authors certify that they have participated sufficiently in the work to take public responsibility for the content.

\section{Availability of Data and Materials}

The data sets used and analyzed during the current study are available from the corresponding author on reasonable request.

\section{References}

1 Fröhlich M, Driessen A, Böhmer A, Nienaber U, Igressa A, Probst C, et al; the TraumaRegister DGU. Is the shock index based classification of hypovolemic shock applicable in multiple injured patients with severe traumatic brain injury? An analysis of the TraumaRegister DGU ${ }^{\circledR}$. Scand J Trauma Resusc Emerg Med. 2016 Dec;24(1):148.

2 Lui CT, Wong OF, Tsui KL, Kam CW, Li SM, Cheng M, et al. Predictive model integrating dynamic parameters for massive blood transfusion in major trauma patients: the Dynamic MBT score. Am J Emerg Med. 2018 Aug;36(8):1444-50.

3 Parks JK, Elliott AC, Gentilello LM, Shafi S. Systemic hypotension is a late marker of shock after trauma: a validation study of Advanced Trauma Life Support principles in a large national sample. Am J Surg. 2006 Dec;192(6):727-31.
4 Siegel JH. The effect of associated injuries, blood loss, and oxygen debt on death and disability in blunt traumatic brain injury: the need for early physiologic predictors of severity. J Neurotrauma. 1995 Aug;12(4): 579-90.

5 Rossaint R, Bouillon B, Cerny V, Coats TJ, Duranteau J, Fernández-Mondéjar E, et al. The European guideline on management of major bleeding and coagulopathy following trauma: fourth edition. Crit Care. 2016 Apr;20:100.

6 Stewart RM, Brasel K, Henry S. Shock. In: Stewart RM, Brasel K, Henry S., editors. Advanced trauma life support student course manual. 10th ed. Chicago: American College of Surgeons, The Committee on Trauma; 2018. Chapter 3, p. 43-61.

7 Varga E, Endre E, Kószó B, Pető Z, Ágoston Z, Gyura E, et al. Advanced Trauma Life
Support (ATLS) in Hungary: the first 10 years. Bull Emerg Trauma. 2016 Jan;4(1):4850.

8 Mutschler M, Paffrath T, Wölfl C, Probst C, Nienaber U, Schipper IB, et al. The ATLS $^{\circledR}$ classification of hypovolaemic shock: a well established teaching tool on the edge? Injury. 2014 Oct; 45 Suppl 3:S358.

9 Kortbeek JB, Al Turki SA, Ali J, Antoine JA, Bouillon B, Brasel K, et al. Advanced trauma life support, 8th edition, the evidence for change. J Trauma. 2008;64:1638-50.

10 Mutschler M, Nienaber U, Brockamp T, Wafaisade $\mathrm{A}$, Wyen $\mathrm{H}$, Peiniger $\mathrm{S}$, et al; TraumaRegister DGU. A critical reappraisal of the ATLS classification of hypovolaemic shock: does it really reflect clinical reality? Resuscitation. 2013 Mar;84(3):309-13. 
11 Mutschler M, Nienaber U, Brockamp T, Wafaisade A, Fabian T, Paffrath T, et al; TraumaRegister DGU. Renaissance of base deficit for the initial assessment of trauma patients: a base deficit-based classification for hypovolemic shock developed on data from 16,305 patients derived from the TraumaRegister $\mathrm{DGU}^{\circledR}$. Crit Care. 2013 Mar; 17(2):R42.

12 Porter JM, Ivatury RR. In search of the optimal end points of resuscitation in trauma patients:areview.JTrauma.1998May;44(5):90814.

13 Guerado E, Medina A, Mata MI, Galvan JM, Bertrand ML. Protocols for massive blood transfusion: when and why, and potential complications. Eur J Trauma Emerg Surg. 2016 Jun;42(3):283-95.

14 Caputo N, Fraser R, Paliga A, Kanter M, Hosford K, Madlinger R. Triage vital signs do not correlate with serum lactate or base deficit, and are less predictive of operative intervention in penetrating trauma patients: a prospective cohort study. Emerg Med J. 2013 Jul;30(7):546-50.

15 Dunne JR, Tracy JK, Scalea TM, Napolitano LM. Lactate and base deficit in trauma: does alcohol or drug use impair their predictive accuracy? J Trauma. 2005 May;58(5):959-66.

16 Ibrahim I, Chor WP, Chue KM, Tan CS, Tan HL, Siddiqui FJ, et al. Is arterial base deficit still a useful prognostic marker in trauma? A systematic review. Am J Emerg Med. 2016 Mar;34(3):626-35.

17 Lam SW, Lingsma HF, van Beeck EF, Leenen LP. Validation of a base deficit-based trauma prediction model and comparison with TRISS and ASCOT. Eur J Trauma Emerg Surg. 2016 Oct;42(5):627-33.

18 Raux M, Le Manach Y, Gauss T, Baumgarten R, Hamada S, Harrois A, et al; TRAUMABASE Group. Comparison of the Prognostic Significance of Initial Blood Lactate and Base Deficit in Trauma Patients. Anesthesiology. 2017 Mar;126(3):522-33.

19 Dunham MP, Sartorius B, Laing GL, Bruce JL, Clarke DL. A comparison of base deficit and vital signs in the early assessment of patients with penetrating trauma in a high burden setting. Injury. 2017 Sep;48(9):1972-7.
20 Herbert HK, Dechert TA, Wolfe L, Aboutanos MB, Malhotra AK, Ivatury RR, et al. Lactate in trauma: a poor predictor of mortality in the setting of alcohol ingestion. Am Surg. 2011 Dec;77(12):1576-9.

21 Gustafson ML, Hollosi S, Chumbe JT, Samanta D, Modak A, Bethea A. The effect of ethanol on lactate and base deficit as predictors of morbidity and mortality in trauma. Am J Emerg Med. 2015 May;33(5):607-13.

22 Davis JW, Kaups KL. Base deficit in the elderly: a marker of severe injury and death. J Trauma. 1998 Nov;45(5):873-7.

23 Shakur H, Roberts I, Bautista R, Caballero J, Coats T, Dewan Y, et al.; CRASH-2 trial collaborators. Effects of tranexamic acid on death, vascular occlusive events, and blood transfusion in trauma patients with significant haemorrhage (CRASH-2): a randomised, placebo-controlled trial. Lancet. 2010 Jul;376(9734):23-32.

24 Kutcher ME, Kornblith LZ, Narayan R, Curd V, Daley AT, Redick BJ, et al. A paradigm shift in trauma resuscitation: evaluation of evolving massive transfusion practices. JAMA Surg. 2013 Sep;148(9):834-40.

25 Hafeez W. Resuscitation. In: Cunningham S, Crain E, Gershel J, editors. Clinical manual of emergency pediatrics. Cambridge: Cambridge University; 2010. p. 1-29.

26 Fleming S, Thompson M, Stevens R, Heneghan C, Plüddemann A, Maconochie I, et al. Normal ranges of heart rate and respiratory rate in children from birth to 18 years of age: a systematic review of observational studies. Lancet. 2011 Mar;377(9770):1011-8.

27 Beloncle F, Meziani F, Lerolle N, Radermacher P, Asfar P. Does vasopressor therapy have an indication in hemorrhagic shock? Ann Intensive Care. 2013 May;3(1):13.

28 Gupta B, Garg N, Ramachandran R. Vasopressors: do they have any role in hemorrhagic shock? J Anaesthesiol Clin Pharmacol. 2017 Jan-Mar;33(1):3-8.

29 Hylands M, Toma A, Beaudoin N, Frenette AJ, D’Aragon F, Belley-Côté É, et al. Early vasopressor use following traumatic injury: a systematic review. BMJ Open. 2017 Nov;7(11):e017559.
30 Mutschler $M$, Nienaber U, Münzberg $M$, Wölfl C, Schoechl H, Paffrath T, et al; TraumaRegister DGU. The Shock Index revisited - a fast guide to transfusion requirement? A retrospective analysis on 21,853 patients derived from the TraumaRegister DGU. Crit Care. 2013 Aug;17(4):R172.

31 Mofidi M, Hasani A, Kianmehr N. Determining the accuracy of base deficit in diagnosis of intra-abdominal injury in patients with blunt abdominal trauma. Am J Emerg Med. 2010 Oct:28(8):933-6.

32 Aukema TS, Hietbrink F, Beenen LF, Leenen LP. Does thoracic injury impair the predictive value of base deficit in trauma patients? Injury. 2010 Apr;41(9):935-7.

33 Bonanno FG. Hemorrhagic shock: the "physiology approach". J Emerg Trauma Shock. 2012 Oct;5(4):285-95.

34 Abou-Khalil B, Scalea TM, Trooskin SZ, Henry SM, Hitchcock R. Hemodynamic responses to shock in young trauma patients: need for invasive monitoring. Crit Care Med. 1994 Apr;22(4):633-9.

35 Guly HR, Bouamra O, Little R, Dark P, Coats $\mathrm{T}$, Driscoll $\mathrm{P}$, et al. Testing the validity of the ATLS classification of hypovolaemic shock. Resuscitation. 2010 Sep;81(9):1142-7.

36 Brasel KJ, Guse C, Gentilello LM, Nirula R. Heart rate: is it truly a vital sign? J Trauma. 2007 Apr;62(4):812-7.

37 Loftus TJ, Efron PA, Moldawer LL, Mohr AM. $\beta$-Blockade use for Traumatic Injuries and Immunomodulation: A Review of Proposed Mechanisms and Clinical Evidence. Shock. 2016 Oct;46(4):341-51.

38 Taniguchi T, Kurita A, Yamamoto K, Inaba $\mathrm{H}$. Effects of carvedilol on mortality and inflammatory responses to severe hemorrhagic shock in rats. Shock. 2009 Sep;32(3):272-5.

39 Bonanno FG. Clinical pathology of the shock syndromes. J Emerg Trauma Shock. 2011 Apr;4(2):233-43.

40 Cooke WH, Salinas J, Convertino VA, Ludwig DA, Hinds D, Duke JH, et al. Heart rate variability and its association with mortality in prehospital trauma patients. J Trauma. 2006 Feb;60(2):363-70.

41 Little RA, Kirkman E, Driscoll P, Hanson J, Mackway-Jones K. Preventable deaths after injury: why are the traditional 'vital' signs poor indicators of blood loss? J Accid Emerg Med. 1995 Mar;12(1):1-14. 